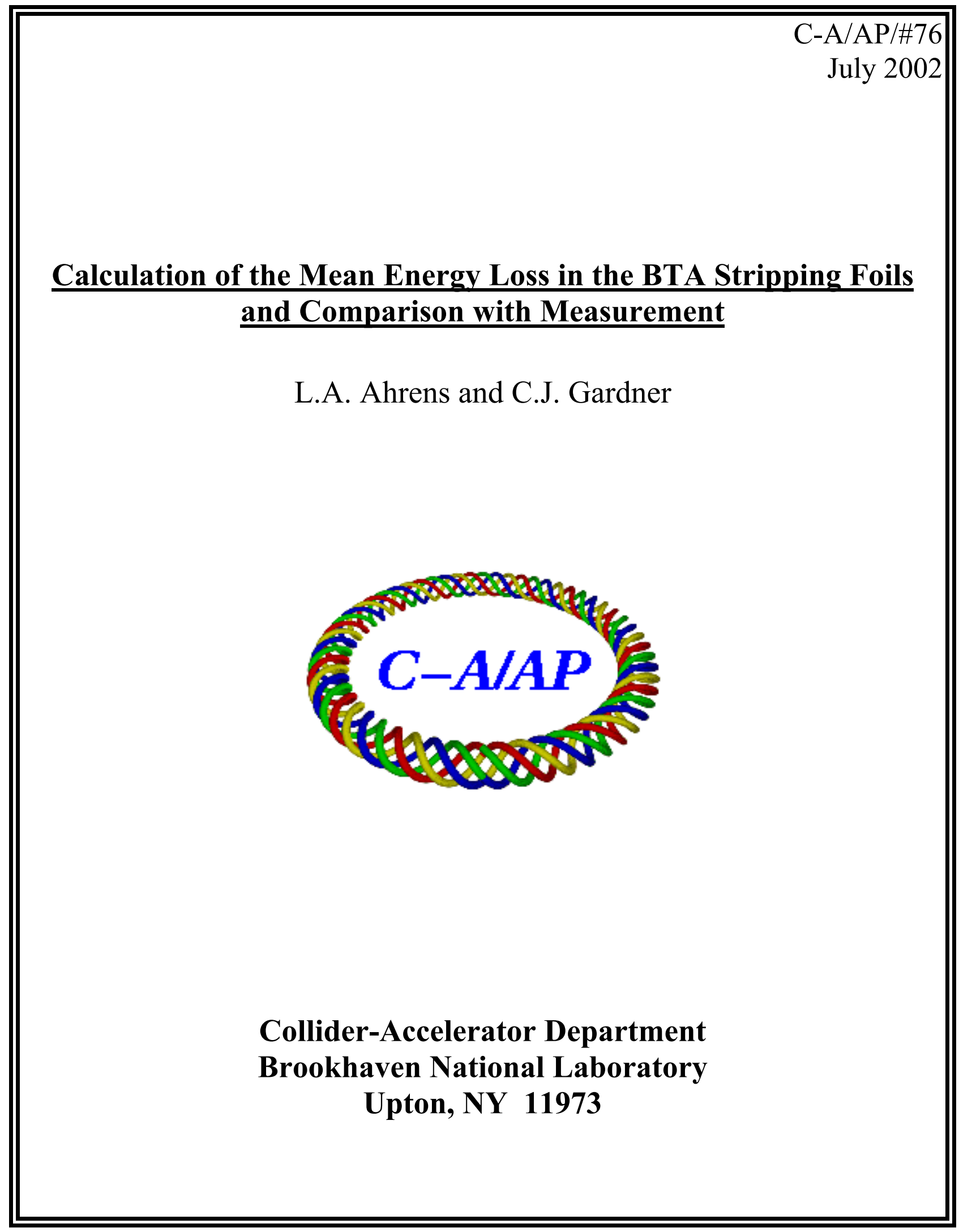




\title{
Calculation of the Mean Energy Loss in the BTA Stripping Foils and Comparison with Measurement
}

\author{
L.A. Ahrens and C.J. Gardner
}

July 1,2002

Following is a calculation of the mean energy lost by various ions in the BTA stripping foils. The calculation is based on the known thickness and density of the foils; the known energy of the ions incident on the foil; and the known energy loss rates for protons in the foil material. The results of the calculation are in good agreement with the measured revolution frequencies at Booster extraction and AGS injection.

\section{Booster Extraction Parameters}

The nominal parameters for various ions at Booster extraction are summarized in Table 1. Here $N$ is the number of nucleons, $p_{b} / N$ is the momentum per nucleon, $W / N$ is the kinetic energy per nucleon, $c \beta_{b}$ is the velocity, and $f_{b}$ is the revolution frequency. In terms of $\beta_{b}$ we have

$$
p_{b}=m_{b} c \beta_{b} \gamma_{b}, \quad W=m_{b} c^{2}\left(\gamma_{b}-1\right), \quad f_{b}=\frac{c \beta_{b}}{2 \pi R_{b}}, \quad \gamma_{b}=\left(1-\beta_{b}^{2}\right)^{-1 / 2}
$$

where $R_{b}=128.4526 / 4$ (meters) is the Booster radius and $m_{b}$ is the mass of the ion in Booster. The ion energy is

$$
E_{b}=m_{b} c^{2} \gamma_{b}=\sqrt{c^{2} p_{b}^{2}+m_{b}^{2} c^{4}}
$$

\section{BTA Stripping Foils}

In the BTA (Booster-To-AGS) transport line, the ions pass through a stripping foil located 0.3706 meters downstream of quadrupole QV3 and 
Table 1: Booster Extraction Parameters

\begin{tabular}{|c|c|c|c|c|c|}
\hline Ion & $N$ & $p_{b} / N(\mathrm{MeV} / \mathrm{c})$ & $W / N(\mathrm{MeV})$ & $\beta_{b}$ & $f_{b}(\mathrm{MHz})$ \\
\hline $\mathrm{Si}^{5+}$ & 28 & 431.3611 & 95.1105 & 0.42053415 & $3.748957 / 6$ \\
\hline $\mathrm{Fe}^{10+}$ & 56 & 431.3611 & 95.1397 & 0.42065119 & $3.750000 / 6$ \\
\hline $\mathrm{Au}^{32+}$ & 197 & 431.7933 & 95.2350 & 0.42065113 & $5.000000 / 8$ \\
\hline $\mathrm{Au}^{32+}$ & 197 & 445.7235 & 101.1721 & 0.43172485 & $3.848719 / 6$ \\
\hline
\end{tabular}

0.9440 meters upstream of quadrupole QH4. At this location, seven foils are mounted on a rotatable circular array of eight equally spaced holders, seven of which contain foils and one which is left empty. Each foil can be inserted into the line by rotating its holder into the beam aperture. If no stripping is desired, the empty holder is rotated into the aperture. The foil material and thicknesses are listed in Table $\mathbf{2}$.

Table 2: BTA Stripping Foils

\begin{tabular}{|c|c|c|c|}
\hline Holder & Foil Material & $\Delta t$ & $\Delta x$ \\
\hline 1 & Empty & Inches & $\mathrm{mg} / \mathrm{cm}^{2}$ \\
\hline 2 & Carbon & .003 & 13.9 \\
3 & Carbon & .004 & 18.5 \\
4 & Carbon & .005 & 23.1 \\
5 & Carbon & .007 & 32.4 \\
6 & Carbon & .010 & 46.2 \\
\hline 7 & Copper & .001 & 22.8 \\
\hline 8 & Copper/Carbon & $.001 / .003$ & $22.8 / 13.9$ \\
\hline
\end{tabular}

Here the holder number is the spreadsheet command that must be issued to insert the corresponding foil into the beam aperture. The thicknesses $\Delta t$ are those quoted by the manufacturer. For the Carbon foils they are 
good to about 0.0001 inches; for the Copper foil they are good to about 10 per cent. According to the manufacturer, the Carbon foils are Graphite with a density $\rho=1.82 \mathrm{~g} / \mathrm{cm}^{3}$. The nominal density of Copper is $\rho=8.96$ $\mathrm{g} / \mathrm{cm}^{3}[1]$. The thickness parameter $\Delta x=\rho \Delta t$ has been calculated assuming these densities.

The choice of materials and thicknesses for the set of seven foils is based on the calculations and measurements of Roser $[2,3,4]$. The carbon foils in holders 3 and 4 have been used for several years to strip gold ions to charge state 77 . The carbon foil in holder 2 is used to fully strip iron and silicon ions. (Until 1997, the carbon foil in holder 6 was used to strip the iron and silicon ions.) The carbon foil in holder 5 and the copper foils in holders 7 and 8 have not been used.

\section{$3 \quad$ Energy Loss Rates in the Foil Material}

As ions from Booster pass through the stripping foil they lose energy due to interaction with the foil material. The mean rate of energy loss is expressed as the derivative $d E / d x$ where $d E$ is the change in energy along a distance $d t=d x / \rho$ of material with density $\rho$. The energy loss rate for a given ion passing through a given material can be calculated from the scaling law

$$
\frac{d E_{I}}{d x}=\frac{Z^{2}}{N} \times \frac{d E_{p}}{d x}
$$

which follows from the Bethe-Bloch equation $[5,6]$ and relates the loss rate of the ion to that of a proton passing through the same material. Here $d E_{I} / d x$ is the mean rate of energy loss per nucleon for an ion with $N$ nucleons, charge $Z$, and velocity $c \beta_{I}$ in the given material. $d E_{p} / d x$ is the mean rate of energy loss for a proton with the same velocity $\left(c \beta_{I}\right)$ in the material.

Thus, to obtain the value of $d E_{I} / d x$ for the ions in Table 1 , we need the value of $d E_{p} / d x$ for protons with velocity $c \beta_{b}$. This can be obtained from the plot of $-d E / d x$ versus $\beta \gamma$ given in Ref. [5]. As shown in the appendix, one finds that for protons in carbon with $\beta \gamma$ between 0.4472 and 0.5 , $d E_{p} / d x$ is given by the approximate expression

$$
-\frac{d E_{p}}{d x}=10^{b}(\beta \gamma)^{m}
$$

where $m=-1.38163$ and $b=0.36224$. The values of $\beta_{b} \gamma_{b}$ for the ions in Table 1 are listed in the third row of Table 3. Substituting these values 
into (4) gives the values of $-d E_{p} / d x$ listed in the fourth row of the Table. The fifth row lists the values of $Z^{2} / N$. Here we assume that the silicon, iron, and gold ions from Booster quickly reach charge states 14, 26, and 77 respectively in the stripping foil. Finally, substituting the values of $d E_{p} / d x$ and $Z^{2} / N$ into (3), we obtain the values of $-d E_{I} / d x$ listed in the sixth row of the Table. The units of $d E / d x$ here are $\mathrm{MeV}$ per $\mathrm{g} / \mathrm{cm}^{2}$.

\section{Mean Energy Lost upon Traversal of a Foil}

The mean energy $\Delta E$ lost upon traversal a foil is given by

$$
\Delta E / N=-\left(d E_{I} / d x\right) \Delta x
$$

where $N$ is the number of nucleons, $-d E_{I} / d x$ is the loss rate from Table 3 and $\Delta x$ is the foil thickness parameter from Table 2 . The values of $\Delta E / N$

obtained for the ions of Table 3 passing through the carbon foils in holders 2 through 6 are listed in Table 4 . (The numbers listed directly below the ion names are the momenta per nucleon in $\mathrm{MeV} / \mathrm{c}$.)

\section{Calculated and Measured Change in AGS Injection Frequency due to Energy Loss in Foil}

The energy of ions injected into AGS is

$$
E=m c^{2} \gamma_{b}-\Delta E
$$

where $m$ is the ion mass in AGS, $\gamma_{b}$ is the value of $\gamma$ at Booster extraction, and $\Delta E$ is the energy lost in the BTA stripping foil. The value of $\beta$ on the AGS injection porch is then

$$
\beta=\sqrt{E^{2}-m^{2} c^{4}} / E
$$

and the revolution frequency is

$$
f=c \beta /(2 \pi R)
$$

where $R=128.4526$ (meters) is the AGS radius. If $\Delta E=0$, then the revolution frequency on the injection porch is

$$
f_{0}=c \beta_{b} /(2 \pi R)
$$


where $\beta_{b}$ is the value of $\beta$ at Booster extraction. The change in the AGS injection frequency due to the energy loss in the foil is then

$$
\Delta=h\left(f_{0}-f\right)
$$

where $h$ is the AGS harmonic number.

Table 5 lists the values of $\Delta$ calculated using the Booster extraction parameters in Table 1 and the values of $\Delta E / N$ from Table 4 . The values of $\Delta$ obtained from measurements of the frequency at extraction in Booster and at injection in AGS are listed as $\Delta_{M}$ in Table 5. The 1995 measurements were done by Hayes [7]; the others were done by Ahrens $[8,9,10]$. These measurements are good to approximately $1 \mathrm{kHz}$. Comparing the numbers in the last two columns of the table, we see that the calculated and measured values of $\Delta$ are in good agreement. Note, however, that the measured values are all lower than the calculated values. This is consistent with the fact that the ions start out in the stripping foil with lower charge states than those assumed in the calculation. According to the scaling law (3), this gives a smaller energy loss rate during the initial part of the ion's passage through the foil.

\section{Appendix}

Energy loss curves for protons in liquid hydrogen, gaseous helium, carbon, aluminium, iron, tin, and lead are shown in Figure 1 which is taken from Ref. [5]. We are interested in the carbon curve (labeled "C") for protons with $\beta \gamma$ between 0.46 and 0.48 . Figure 2 shows a magnified view of this region. Here we see that the carbon curve crosses the line $-d E_{p} / d x=7$ at a point halfway between $\beta \gamma=0.4$ and $\beta \gamma=0.5$. Since the horizontal scale is logarithmic, the value of $\beta \gamma$ for this point is given by

$$
\log (\beta \gamma)=\frac{1}{2}(\log 0.4+\log 0.5)
$$

which gives

$$
\beta \gamma=\sqrt{0.4} \sqrt{0.5}=0.4472136
$$

We also see that the carbon curve crosses the line $-d E_{p} / d x=6$ at the point where $\beta \gamma=0.5$. Between these two values of $\beta \gamma$, the curve is approximately linear, and, since the horizontal and vertical scales are logarithmic, we have

$$
\log \left(-\frac{d E_{p}}{d x}\right)=m \log (\beta \gamma)+b
$$


where

$$
m=\frac{\log 6-\log 7}{\log 0.5-\log 0.4472136}=-1.38163
$$

and

$$
b=\log 6-m \log 0.5=0.36224
$$

Thus, for protons in carbon with $\beta \gamma$ between 0.4472136 and 0.5 we have

$$
-\frac{d E_{p}}{d x}=10^{b}(\beta \gamma)^{m}
$$

\section{References}

[1] D.E. Groom, et al., The European Physical Journal C 15, 80 (2000)

[2] T. Roser, "BTA Stripping Foil Studies", AGS/AD/Tech. Note No. 367, October 2, 1992.

[3] T. Roser, "Stripping Efficiencies for $277 \mathrm{MeV} / \mathrm{amu}$ Gold Beam on Copper Foils", Proceedings of the 1993 Particle Accelerator Conference, Washington, D.C., May 17-20, 1993, pp.3207-3209.

[4] L. Ahrens, H.C. Hseuh, and T. Roser, "Charge Exchange Studies with Gold Ions at the Brookhaven Booster and AGS", Fourth European Particle Accelerator Conference, London, England, June 27-July 1, 1994, pp. 2441-2443.

[5] D.E. Groom, et al., The European Physical Journal C 15, 163-173 (2000). This material also may be found at http://pdg.lbl.gov/ contents_sports.html, "Passage of particles through matter (2000v)".

[6] J.D. Jackson, "Classical Electrodynamics", Third Edition, John Wiley \& Sons, Inc., New York, 1998, Chapter 13

[7] T. Hayes, verbal communication, 1995

[8] L.A. Ahrens, Logbook AG.303.2.9701, October 29, 1996, p. 97

[9] L.A. Ahrens, Logbook AG.303.2.0001, February 1, 2000, pp. 30-31

[10] L.A. Ahrens, Logbook AG.303.2.0102, May 2, 2001, pp. 47-49 
Table 3: Calculated Rates of Energy Loss in Carbon

\begin{tabular}{|c|c|c|c|c|}
\hline Ion & $\mathrm{Si}^{14+}$ & $\mathrm{Fe}^{26+}$ & $\mathrm{Au}^{77+}$ & $\mathrm{Au}^{77+}$ \\
\hline$c p_{b}(\mathrm{MeV})$ & 431.4 & 431.4 & 431.8 & 445.7 \\
\hline$\beta_{b} \gamma_{b}$ & 0.4635 & 0.4637 & 0.4637 & 0.4786 \\
\hline$-d E_{p} / d x$ & 6.662 & 6.659 & 6.659 & 6.374 \\
\hline$Z^{2} / N$ & $14^{2} / 28$ & $26^{2} / 56$ & $77^{2} / 197$ & $77^{2} / 197$ \\
\hline$-d E_{I} / d x$ & 46.63 & 80.38 & 200.4 & 191.8 \\
\hline
\end{tabular}

Table 4: Calculated Energy Loss in Carbon Foils

\begin{tabular}{|c|c|c|c|c|c|}
\hline Foil & Thickness & $\mathrm{Si}^{14+}$ & $\mathrm{Fe}^{26+}$ & $\mathrm{Au}^{77+}$ & $\mathrm{Au}^{77+}$ \\
Holder & $\Delta x$ & 431.4 & 431.4 & 431.8 & 445.7 \\
\cline { 3 - 6 } Number & $\left(\mathrm{mg} / \mathrm{cm}^{2}\right)$ & \multicolumn{4}{|c|}{$\Delta E / \mathrm{MeV}$ per Nucleon $)$} \\
\hline 2 & 13.9 & 0.65 & 1.12 & 2.79 & 2.67 \\
3 & 18.5 & 0.86 & 1.49 & 3.71 & 3.55 \\
4 & 23.1 & 1.08 & 1.86 & 4.63 & 4.43 \\
5 & 32.4 & 1.51 & 2.60 & 6.49 & 6.21 \\
6 & 46.2 & 2.15 & 3.71 & 9.26 & 8.86 \\
\hline
\end{tabular}

Table 5: Calculated and Measured Change in AGS Injection Frequency due to Energy Loss in the BTA Foil.

\begin{tabular}{|c|c|c|c|c|c|c|c|c|}
\hline Year & Ion & $\begin{array}{c}\text { Foil } \\
\text { Holder }\end{array}$ & $\begin{array}{c}\Delta E / N \\
(\mathrm{MeV})\end{array}$ & $h$ & $\begin{array}{c}h f_{0} \\
(\mathrm{MHz})\end{array}$ & $\begin{array}{c}h f \\
(\mathrm{MHz})\end{array}$ & $\begin{array}{c}\Delta \\
(\mathrm{kHz})\end{array}$ & $\begin{array}{c}\Delta_{M} \\
(\mathrm{kHz})\end{array}$ \\
\cline { 3 - 9 } 1995 & $\mathrm{Au}^{77+}$ & 3 & 3.71 & 12 & 1.8750 & 1.8430 & 32.0 & 29.5 \\
1996 & $\mathrm{Fe}^{26+}$ & 6 & 3.71 & 12 & 1.8750 & 1.8430 & 32.0 & 30.4 \\
2000 & $\mathrm{Au}^{77+}$ & 3 & 3.55 & 24 & 3.8487 & 3.7902 & 58.5 & 57.0 \\
2001 & $\mathrm{Au}^{77+}$ & 3 & 3.55 & 24 & 3.8487 & 3.7902 & 58.5 & 57.0 \\
\hline
\end{tabular}




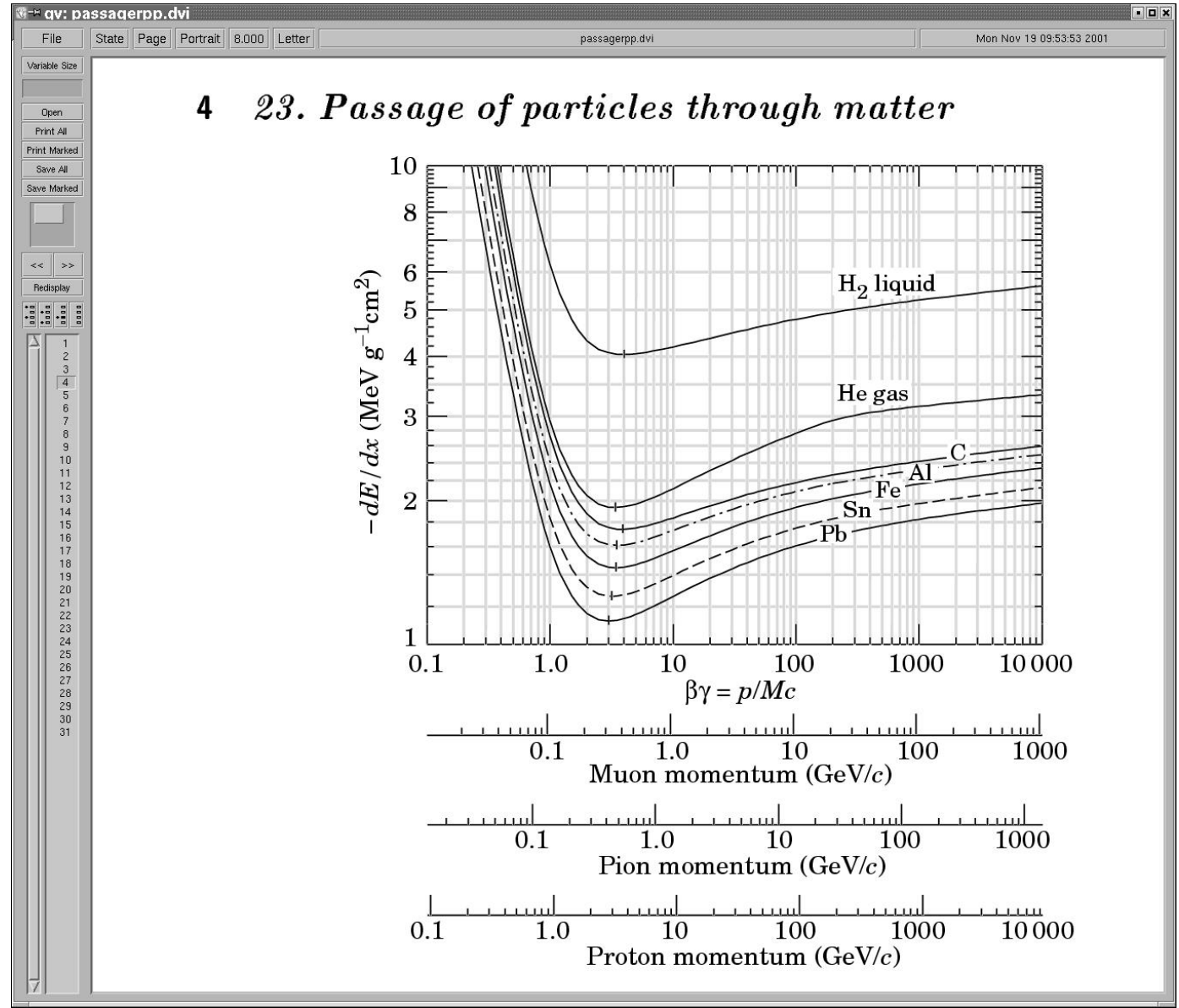

Figure 1: $-d E / d x$ versus momentum for protons passing through liquid hydrogen, gaseous helium, carbon, aluminium, iron, tin, and lead [5]. 


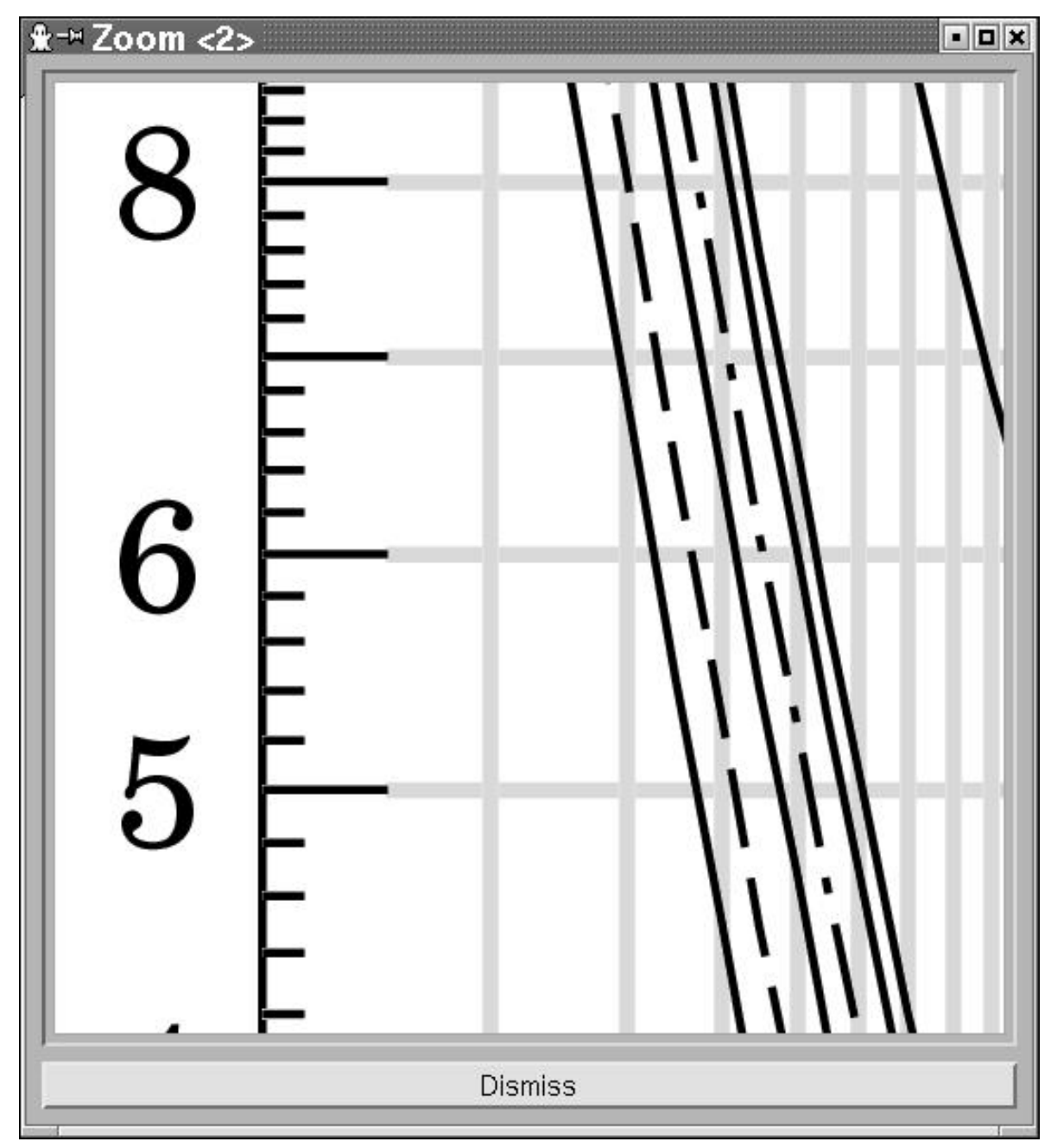

Figure 2: Magnified view of Figure 1. 